\title{
Nitric oxide in liver fibrosis: The role of inducible nitric oxide synthase
}

\author{
Yasuko Iwakiri \\ Department of Internal Medicine, Section of Digestive Diseases, Yale University School of Medicine, New Haven, CT, USA.
}

The inducible form of nitric oxide synthase (iNOS) is expressed in hepatic cells in pathological conditions. Its induction is involved in the development of liver fibrosis, and thus iNOS could be a therapeutic target for liver fibrosis. This review summarizes the role of iNOS in liver fibrosis, focusing on 1) iNOS biology, 2) iNOS-expressing liver cells, 3) iNOS-related therapeutic strategies, and 4) future directions. (Clin Mol Hepatol 2015;21:319-325)

Keywords: iNOS; NO; Liver sinusoidal endothelial cell; Hepatic stellate cell; Kupffer cell

\section{INTRODUCTION}

Nitric oxide (NO) plays important roles in hepatic physiology and pathophysiology. $\mathrm{NO}$ is generated as a by-product of the oxidation of L-arginine to citrulline ${ }^{2}$ by the action of three isoforms of NO synthases (NOSs): neuronal NOS (nNOS or NOS1), inducible NOS (iNOS or NOS2), and endothelial NOS (eNOS or NOS3). nNOS and eNOS are constitutively expressed. iNOS expression is absent in resting cells but is induced by immunological stimuli, such as bacterial lipopolysaccharide (LPS) and inflammatory cytokines. ${ }^{3}$ In the liver, small amounts of NO generated by eNOS are thought to be important for liver homeostasis and protective against pathological conditions. In contrast, large amounts of NO generated by
iNOS are implicated in the etiologies of many liver diseases, including liver fibrosis. The role of nNOS in liver biology is not well known.

This review article will summarize the role of iNOS in liver fibrosis, addressing 1) iNOS biology, 2) iNOS-expressing liver cells, 3) iNOS-related therapeutic strategies, and 4) future directions.

\section{iNOS BIOLOGY}

\section{Mode of action of NO}

NO can regulate biological processes by virtue of its highly reac-

\footnotetext{
Abbreviations:

AP-1, activator protein-1; $\mathrm{BH} 4$, tetrahydrobiopterin; $\mathrm{CGMP}$, cyclic guanosine monophosphate; ECMs, extracellular matrix proteins; $\mathrm{ENOS}$, endothelial nitric oxide synthase; ER- $\alpha$ and $-\beta$, estrogen receptor- $\alpha$ and $-\beta$; FGF-2, fibroblast growth factor-2; GR- $\alpha$ and $-\beta$, glucocorticoid receptor- $\alpha$ and $-\beta$; GTPCH, GTP cyclohydrolase 1; HSCs, hepatic stellate cells; interleukin-6, IL-6; iNOS, inducible nitric oxide synthase; IRF-1, interferon regulatory factor-1; LPS, lipopolysaccharide; GC, guanylyl cyclase; LSECs, liver sinusoidal endothelial cells; MMP-9, matrix metalloprotein-9; NAFLD, non-alcoholic fatty liver disease; $\mathrm{NASH}$, non-alcoholic steatohepatitis; NFk $\beta$, nuclear factor-kappa $\beta ;$ nNOS, neuronal nitric oxide synthase; NO, nitric oxide; NOS, nitric oxide synthase; ONOO-, peroxynitrite; PAI-1, plasminogen activator inhibitor-1; PDGF-A and $-B$, platelet-derived growth factor-A and -B; PKG, CGMP-dependent protein kinase; RNS, reactive nitrogen species; SNPs, single nucleotide polymorphisms; miRNA, microRNA; snRNA, small nuclear RNAs; STAT-1a, signal transducer and activator of transcription-1a; STAT-3, signal transducer and activator of transcription-3; TCF-11, transcription factor 11; TCF-4, T-cell factor 4; TGF- $\beta$; transforming growth factor- $\beta$; TNF- $\alpha$, tumor necrosis factor- $\alpha$
}

\section{Corresponding author: Yasuko Iwakiri}

Department of Internal Medicine, Section of Digestive Diseases, Yale University School of Medicine, 333 Cedar street, New Haven, CT 06520, USA.

Tel: +1-203-785-6204, Fax: +1-203-785-7273

Email: yasuko.iwakiri@yale.edu 
tive nature. NO can bind to a wide range of molecules, such as free radicals, metal centers of enzymes [e.g., guanylyl cyclase (GC) and cytochrome $\mathrm{C}$ oxidase], tyrosine or cysteine residues of proteins, guanine nucleotides, and polyunsaturated fatty acids.'

In the most renowned mode of NO action, NO activates GC by binding to its metal center (i.e., ferrous heme iron). This produces cyclic guanosine monophosphate (cGMP), which then binds to phosphodiesterases or cGMP-dependent protein kinases (PKGs) and induces a cascade of events that facilitate various biological processes, such as vasodilation, platelet aggregation, and neurotransmission. ${ }^{4}$ iNOS-derived NO exerts its effects through these various pathways and is involved in the development and maintenance of many liver diseases.

\section{Regulation of iNOS expression by transcription factors}

Due to its inducible nature, the regulation of iNOS transcription is considered the most important step in the control of iNOS activity, as opposed to constitutively expressed eNOS and nNOS, the regulation of which is more heavily governed by post-translational modifications and protein-protein interactions than by mRNA expression. The most studied pathway in the liver is the activation of the transcription factor, nuclear factor-kappa $\beta$ (NF- $\kappa \beta$ ), which can bind to the iNOS promoter and induce iNOS expression. In addition, various transcription factors have been shown to regulate iNOS expression. Those include signal transducer and activator of transcription-1 $\alpha$ (STAT-1 $\alpha$ ), interferon regulatory factor-1 (IRF-1), activator protein-1 (AP-1), octamer transcription factors, cAMP-induced transcription factors, T-cell factor 4 (TCF-4), transcription factor 11 (TCF-11), epidermal growth factor receptor, signal transducer and activator of transcription-3 (STAT-3), nuclear receptors, glucocorticoid receptor- $\alpha$ and $-\beta$ (GR- $\alpha$ and $-\beta$ ), and estrogen receptor- $\alpha$ and $-\beta$ (ER- $\alpha$ and $-\beta$ ). Furthermore, signaling pathways that stimulate these transcription factors appear to differ, depending on cell type and species. ${ }^{5}$ Understanding these differences in iNOS inducers and their related pathways could be a key to the modulation of iNOS induction. In addition to transcriptional regulation, the control of iNOS mRNA stability, mRNA translation, and protein stability plays a critical role in iNOS induction. ${ }^{6}$

\section{Regulation of iNOS enzymatic activity}

iNOS activity is dependent on the availability of its substrate, arginine, and is influenced by the activity of arginase, which cata- lyzes the conversion of L-arginine to L-ornithine and urea, thereby decreasing the availability of L-arginine for iNOS activity. ${ }^{7}$ Tetrahydrobiopterin $\left(\mathrm{BH}_{4}\right)$ is an essential cofactor for iNOS activity. ${ }^{8,9} \mathrm{Be}-$ cause homodimerization of iNOS is required for its activity and $\mathrm{BH} 4$ is essential for this process, the mechanism regulating $\mathrm{BH}_{4}$ synthesis and consumption can also regulate iNOS activity. ${ }^{8-11}$ GTP cyclohydrolase 1 (GTPCH) is an essential enzyme in the biogenesis of $\mathrm{BH}_{4}$. Deletion of this enzyme in macrophages blocked NO synthesis in response to LPS and IFN $\gamma$ stimulation, despite the presence of iNOS protein. In addition, a lack of $\mathrm{BH}_{4}$ biogenesis resulted in increased production of reactive oxygen species (ROS) due to the uncoupling of iNOS, which is mediated by $\mathrm{BH}_{4}$. However, these events were reversible because the administration of sepiapterin, a precursor of $\mathrm{BH}_{4}$, could restore $\mathrm{BH}_{4}$, NO production, and the cellular redox state. ${ }^{12}$

Inhibitory proteins of iNOS activity have been identified. In murine macrophages, a $110-\mathrm{kDa}$ protein, called NAP110, directly interacted with the amino terminus of INOS and prevented its dimerization, thereby inhibiting iNOS activity. ${ }^{13}$ iNOS protein synthesizes NO continuously until the enzyme is degraded. ${ }^{14,15}$ Therefore, factors affecting iNOS protein degradation could regulate iNOS activity and NO production.

\section{iNOS AND LIVER FIBROSIS}

Liver fibrosis is a consequence of a wide range of liver injuries caused by hepatitis virus, drug, toxin, bile acid accumulation, autoimmune diseases, obesity/metabolic syndrome, and alcohol. ${ }^{1}$ iNOS induction is associated with liver fibrosis of diverse etiology.'

The role of iNOS has been studied using iNOS knockout mice and iNOS-specific or NOS inhibitors in vivo and in vitro. iNOS gene deletion resulted in reduced liver fibrosis. ${ }^{16,17}$ iNOS KO mice fed a high-cholesterol diet for 6 weeks exhibited significant reductions in hepatic fibrosis and expression of inflammatory cytokines, including transforming growth factor-beta (TGF $\beta$ ) and tumor necrosis factor-alpha (TNF $\alpha)^{1 .}{ }^{16}$ In addition, iNOS deletion decreased hypoxia-inducible factor-1alpha (HIF1 $\alpha$ ) levels and HIF1 $\alpha$-associated gene expression, including that of platelet-derived growth factor (PDGF)A, PDGF-B, fibroblast growth factor-2 (FGF-2), and plasminogen activator inhibitor-1 (PAI-1). A similar reduction in high-cholesterol diet-induced hepatic fibrosis was observed in mice provided with an iNOS-specific inhibitor (PBIT). Taken together, both genetic deletion and pharmacological inhibition of iNOS resulted in decreased hepatic fibrosis, suggesting a role of INOS in the development of 
liver fibrosis.

To further understand its role in liver fibrosis, cell-specific roles of iNOS have to be examined. The liver contains parenchymal cells, such as hepatocytes and cholangiocytes, and non-parenchymal cells, including Kupffer cells, liver sinusoidal endothelial cells (LSECs), and hepatic stellate cells (HSCs) as the major cells. Other smaller populations of liver cells, such as portal fibroblasts, dendritic cells, T-lymphocytes, and B-lymphocytes, are also involved in hepatic homeostasis and pathophysiology. iNOS is induced in almost all hepatic cells in response to various stimuli, but Kupffer cells would be the most studied hepatic cells for the regulation of iNOS among other liver cells. Cell-specific roles of iNOS-derived NO in liver fibrosis will be addressed below.

\section{Kupffer cells}

Activated Kupffer cells produce a wide variety of proinflammatory cytokines, such as TNF $\alpha$, IL-6, and TGF $\beta$, which then activate $\mathrm{HSC}$, leading to the production of extracellular matrix (collagen fibers) and thereby, liver fibrosis. In addition, activated Kupffer cells produce large amounts of NO through an induction of INOS. NO reacts with ROS to form reactive nitrogen species (RNS), such as highly reactive and toxic peroxynitrite $\left(\mathrm{ONOO}^{-}\right)$. Kupffer cells are the primary source of $\mathrm{ONOO}^{-}$in the liver. These inflammatory mediators (i.e., ROS, RNS, and $\mathrm{ONOO}^{-}$) can also activate $\mathrm{HSCS}$. Kupffer cells are activated by a wide range of stimuli, such as the endotoxin LPS in the blood and the inflammatory cytokines mentioned above. Generally, high levels of glutathione (GSH), an antioxidant protein, scavenge $\mathrm{ONOO}^{-}$and prevent $\mathrm{HSCs}$ from activation. However, conditions that deplete GSH and/or other antioxidant molecules may increase the risk of $\mathrm{ONOO}^{-}$-induced liver damage and HSC activation, facilitating liver fibrosis. For example, GSH depletion has been demonstrated in alcoholic liver disease. ${ }^{18-20}$ The molecular mechanism by which $\mathrm{ONOO}^{-}$causes HSC activation remains largely unknown.

An antifibrotic effect of NO was also reported, where NO caused apoptosis of myofibroblasts, thereby reducing liver fibrosis. ${ }^{21}$ This increase in apoptosis resulted from mitochondrial dysfunction caused by the penetration of NO and was independent of caspase pathways. How these opposite effects of NO on liver fibrosis are exerted remains to be elucidated.

\section{Liver sinusoidal endothelial cells}

LSECs play an important role in the transfer of nutrients, lipids, and lipoproteins. Under normal conditions, LSECs have fenestrae and lack basement membranes. ${ }^{22}$ However, under pathological conditions, LSECs lose fenestrae and start to form basement membranes. ${ }^{23}$ This pathological transformation of the LSEC phenotype is known as "capillarization." LSEC capillarization occurs earlier than fibrotic development in various liver diseases, including non-alcoholic fatty liver disease (NAFLD)/non-alcoholic steatohepatitis (NASH) $)^{24,25}$ and in experimental models of liver fibrosis. ${ }^{24-26}$ LSEC capillarization appears to facilitate HSC activation. ${ }^{27}$ Upregulation of INOS in LSECs was associated with LSEC capillarization. ${ }^{24,28}$ Because cytokines and LPS can induce iNOS expression in LSECS, ${ }^{29}$ increased iNOS expression may contribute to LSEC capillarization and subsequently, hepatic fibrosis. However, the mechanism by which increased iNOS expression leads to LSEC capillarization is unknown.

NO derived from iNOS was implicated in LSEC dysfunction. ${ }^{30}$ However, what functions of LSECS are influenced by iNOS and how those influences are exerted are largely unknown. Further investigations are needed to determine this aspect.

\section{Hepatic stellate cells}

HSCs are known as the main contributor to liver fibrosis. In response to profibrotic stimuli, quiescent HSCs become activated and transformed to myofibroblasts, which produce collagen and other extracellular matrix proteins (ECMs), leading to liver fibrosis. ${ }^{31,32}$ iNOS inhibition in HSCs has been implicated in antifibrosis. ${ }^{16,17}$ iNOS and NO donors enhanced matrix metalloprotein-9 (MMP-9) expression and activation in HSCs in vivo and in vitro. ${ }^{16}$ HSCs are an important source of MMP-9 during hepatic fibrogenesis. ${ }^{16,33}$ MMPs play important roles in degradation and remodeling of ECM components under both physiological and pathophysiological conditions. ${ }^{16,34}$ While the role of MMP-9 in liver fibrogenesis has not been fully elucidated, a study using MMP-9 knockout mice showed reduced liver fibrosis following hepatic injury, ${ }^{35}$ suggesting a contribution of MMP-9 to liver fibrosis. It has been postulated that MMP-mediated degradation of ECMs in the space of Disse is essential for fibrotic activation of HSCs. Because particular ECM components keep HSCs quiescent in normal liver, MMP-mediated ECM degradation may change ECM components, leading to the activation of HSCs. ${ }^{36}$ iNOS knockout mice and mice administered an iNOS-specific inhibitor (PBIT) showed a significant decrease in liver fibrosis in a NASH model, which was accompanied by decreased MMP-9 activation, compared with WT mice, suggesting a positive link among iNOS, MMP-9, and liver fi- 
brosis. $^{16}$

A complex interplay between hepatic cells contributes to hepatic fibrosis. iNOS is induced in almost all liver cells but paracrine regulation of NO between neighboring cells is largely unknown. Studies using mice with cell-specific iNOS deletion may aid in the exploration of this important area.

\section{iNOS-RELATED THERAPEUTIC STRATEGIES}

Experimental and clinical studies have shown iNOS as a potential therapeutic target for liver fibrosis. The potential of iNOS inhibitors and gene therapies will be briefly discussed.

\section{1. iNOS inhibitors}

Because of the proinflammatory nature of iNOS, its inhibition has been considered as a therapeutic strategy for several disease conditions, including septic shock and asthma. ${ }^{37,38}$ However, these trials were not successful. This may be due in part to global inhibition of iNOS and/or the timing of administration of iNOS inhibitors. Because the roles of iNOS may differ according to cell or tissue type or stage of disease, these aspects should be thoroughly examined for therapeutic purposes.

iNOS inhibitors have not yet been tested in human liver fibrosis. In experimental studies, however, iNOS inhibition or gene deletion prevented liver tissue damage and fibrosis. ${ }^{16}$ Blocking iNOS by oral administration of the inhibitor RF260330 reduced fibrosis in rats by inhibiting TGF $\beta 1$ production. ${ }^{39}$ Thus, iNOS could be a therapeutic target for the treatment of liver fibrosis. However, given that almost all liver cells express iNOS, understanding cell-specific roles of iNOS expression in fibrosis would be important for the development of iNOS targeting. Furthermore, the regulation of iNOS induction as well as its biological consequences may differ according to the stage of fibrosis. Knowledge regarding these aspects would help researchers to develop effective therapeutic strategies with minimal off-target effects.

\section{2. iNOS polymorphism}

Several single nucleotide polymorphisms (SNPs) have been found in the human iNOS promoter, ${ }^{6,40,41}$ and their association with some pathological conditions, such as malaria, ${ }^{42}$ atopic diseases, ${ }^{43}$ multiple sclerosis, ${ }^{44}$ and hypertension, ${ }^{45}$ has been suggested.
iNOS SNPs may also be related to the susceptibility to liver fibrosis. One study ${ }^{46}$ investigated iNOS SNPs in NAFLD patients (a total of 115 patients with NAFLD and 435 healthy control subjects) and found a significant increase in the fibrosis index in patients with the $T$ allele of the iNOS SNP rs1060822 ( $P=0.0465$, Kruskal-Wallis test), suggesting that the iNOS SNPs influence liver fibrosis associated with NAFLD. Therefore, targeting these gene mutations may also represent a potential therapeutic strategy.

\section{FUTURE DIRECTIONS}

\section{Role of iNOS in lymphocytes}

Compared with Kupffer cells (or macrophages), iNOS regulation in other immune cells, such as dendritic cells and B and T lymphocytes, has been less thoroughly studied. iNOS induction in T cells is mediated by lymphoid stromal cells (including lymphatic endothelial cells and fibroblast reticular cells). Given the recent studies showing the importance of these lymphocytes for liver fibrosis, ${ }^{47}$ it would be interesting to study the role of INOS in T lymphocytes in relation to the myofibroblast population during hepatic fibrogenesis.

\section{Regulation of iNOS expression by non-coding RNAs}

A non-coding RNA is a functional RNA that does not encode a protein. The majority of the genome is thought to be transcribed into non-coding RNAs, most of which are spliced and/or processed into smaller RNAs. These non-coding RNAs include microRNA (miRNAs), small nuclear RNAs (snRNAs, involved in splicing), and small nucleolar RNAs (snoRNAs, involved in the modification of ribosomal RNAs) as well as other classes of unidentified small regulatory RNAs and longer RNAs. Non-coding RNAs have been recognized as increasingly important for the regulation of the fate of RNAs.

A miRNA is a small, non-coding, single-stranded RNA molecule that consists of about 22 nucleotides and is found in plants, animals, and some viruses. The function of miRNAs includes silencing and post-transcriptional regulation of gene expression by binding to $3^{\prime}$-UTR sequences of target RNAs. At least 6 miRNAs have been identified that regulate iNOS expression, directly or indirectly. These include miR939, 146a, 26a, and 34b, which downregulate iNOS, and miR-155 and 27b, which participate in its upregulation. ${ }^{48}$ The role of these miRNAs in the regulation of hepatic iNOS expression has not yet been explored. The identification of 
cell-specific miRNAs could be important for cell-targeted blockage of iNOS expression, which may contribute to decreased hepatic injury and fibrosis.

\section{Post-translational modifications of proteins by NO}

By producing large amounts of NO, iNOS could modulate protein function through the various post-translational modifications mentioned above. For example, NO can bind to cysteine residues of specific proteins (i.e., S-nitrosylation) and alter their function, thereby facilitating specific biological processes. ${ }^{49-52}$ It is not known what proteins are the targets for S-nitrosylation in HSCS. The identification of potential targets for S-nitrosylation may help us to understand NO-mediated regulation of HSC function. Thus, proteomic analysis of target proteins for NO-mediated post-translational modifications could advance our knowledge of NO-mediated regulation of liver fibrosis.

\section{CONCLUSION}

Given the beneficial roles of iNOS inhibition and gene deletion in liver fibrosis observed in experimental models, iNOS is likely a potential therapeutic target for liver fibrosis. However, iNOS is expressed in almost all liver cells and its role may differ among liver cell types. Therefore, the determination of the cell-specific roles of iNOS in liver fibrosis and the development of a cell-specific delivery system for iNOS inhibitors may warrant the successful development of antifibrotic therapeutic strategies.

\section{Acknowledgements}

This work was supported by NIH grants R01 DK082600, P30 DK045735 and R21AA023599.

\section{Conflicts of Interest}

I have no financial interest in or financial conflicts with the subject matter or materials discussed in this manuscript.

\section{REFERENCES}

1. Iwakiri Y, Kim MY. Nitric oxide in liver diseases. Trends Pharmacol Sci 2015;36:526-536.

2. Schmidt HH, Murad F. Purification and characterization of a human
NO synthase. Biochem Biophys Res Commun 1991;181:1372-1377.

3. Nathan C. Nitric oxide as a secretory product of mammalian cells. FASEB J 1992;6:3051-3064.

4. Derbyshire ER, Marletta MA. Structure and regulation of soluble guanylate cyclase. Annu Rev Biochem 2012;81:533-559.

5. Kleinert H, Pautz A, Linker K, Schwarz PM. Regulation of the expression of inducible nitric oxide synthase. Eur J Pharmacol 2004;500:255-266.

6. Pautz A, Art J, Hahn S, Nowag S, Voss C, Kleinert H. Regulation of the expression of inducible nitric oxide synthase. Nitric Oxide 2010;23:75-93.

7. Mori M, Gotoh T. Regulation of nitric oxide production by arginine metabolic enzymes. Biochem Biophys Res Commun 2000;275:715-719.

8. Förstermann U, Gath I, Schwarz P, Closs El, Kleinert H. Isoforms of nitric oxide synthase. Properties, cellular distribution and expressional control. Biochem Pharmacol 1995;50:1321-1332.

9. Tzeng E, Billiar TR, Robbins PD, Loftus M, Stuehr DJ. Expression of human inducible nitric oxide synthase in a tetrahydrobiopterin (H4B)deficient cell line: $H 4 B$ promotes assembly of enzyme subunits into an active dimer. Proc Natl Acad Sci U S A 1995;92:11771-11775.

10. Mühl H, Pfeilschifter J. Tetrahydrobiopterin is a limiting factor of nitric oxide generation in interleukin 1 beta-stimulated rat glomerular mesangial cells. Kidney Int 1994;46:1302-1306.

11. Ionova IA, Vásquez-Vivar J, Whitsett J, Herrnreiter A, Medhora M, Cooley BC, et al. Deficient $\mathrm{BH} 4$ production via de novo and salvage pathways regulates NO responses to cytokines in adult cardiac myocytes. Am J Physiol Heart Circ Physiol 2008;295:H2178-H2187.

12. McNeill E, Crabtree MJ, Sahgal N, Patel J, Chuaiphichai S, Iqbal AJ, et al. Regulation of iNOS function and cellular redox state by macrophage Gch1 reveals specific requirements for tetrahydrobiopterin in NRF2 activation. Free Radic Biol Med 2015;79:206-216.

13. Ratovitski EA, Bao C, Quick RA, McMillan A, Kozlovsky C, Lowenstein CJ. An inducible nitric-oxide synthase (NOS)-associated protein inhibits NOS dimerization and activity. J Biol Chem 1999;274:3025030257.

14. MacMicking J, Xie QW, Nathan C. Nitric oxide and macrophage function. Annu Rev Immunol 1997;15:323-350.

15. Geller DA, Billiar TR. Molecular biology of nitric oxide synthases. Cancer Metastasis Rev 1998;17:7-23.

16. Anavi S, Eisenberg-Bord M, Hahn-Obercyger M, Genin O, Pines M, Tirosh 0 . The role of iNOS in cholesterol-induced liver fibrosis. Lab Invest 2015;95:914-924.

17. Aram G, Potter JJ, Liu X, Torbenson MS, Mezey E. Lack of inducible nitric oxide synthase leads to increased hepatic apoptosis and decreased fibrosis in mice after chronic carbon tetrachloride administration. Hepatology 2008;47:2051-2058.

18. Fernández-Checa JC. Alcohol-induced liver disease: when fat and oxidative stress meet. Ann Hepatol 2003;2:69-75. 
19. Bai J, Cederbaum Al. Overexpression of CYP2E1 in mitochondria sensitizes HepG2 cells to the toxicity caused by depletion of glutathione. J Biol Chem 2006;281:5128-5136.

20. Garcia-Ruiz C, Fernandez-Checa JC. Mitochondrial glutathione: hepatocellular survival-death switch. J Gastroenterol Hepatol 2006;21(Suppl 3):S3-S6.

21. Langer DA, Das A, Semela D, Kang-Decker N, Hendrickson H, Bronk $S F$, et al. Nitric oxide promotes caspase-independent hepatic stellate cell apoptosis through the generation of reactive oxygen species. Hepatology 2008;47:1983-1993.

22. Wisse E, De Zanger RB, Jacobs R, McCuskey RS. Scanning electron microscope observations on the structure of portal veins, sinusoids and central veins in rat liver. Scan Electron Microsc 1983;(Pt 3):14411452.

23. Braet $F$, Wisse E. Structural and functional aspects of liver sinusoidal endothelial cell fenestrae: a review. Comp Hepatol 2002;1:1.

24. Xie G, Choi SS, Syn WK, Michelotti GA, Swiderska M, Karaca G, et al. Hedgehog signalling regulates liver sinusoidal endothelial cell capillarisation. Gut 2013;62:299-309.

25. Lee YA, Wallace MC, Friedman SL. Pathobiology of liver fibrosis: a translational success story. Gut 2015;64:830-841.

26. Miyao M, Kotani $H$, Ishida $T$, Kawai $C$, Manabe $S$, Abiru $H$, et al. Pivotal role of liver sinusoidal endothelial cells in NAFLD/NASH progression. Lab Invest 2015;95:1130-1144.

27. Xie G, Wang X, Wang L, Wang L, Atkinson RD, Kanel GC, et al. Role of differentiation of liver sinusoidal endothelial cells in progression and regression of hepatic fibrosis in rats. Gastroenterology 2012;142:918-927. e6.

28. Witek RP, Yang L, Liu R, Jung Y, Omenetti A, Syn WK, et al. Liver cell-derived microparticles activate hedgehog signaling and alter gene expression in hepatic endothelial cells. Gastroenterology 2009;136:320-330. e2.

29. Rockey DC, Chung JJ. Regulation of inducible nitric oxide synthase in hepatic sinusoidal endothelial cells. Am J Physiol 1996;271:G260G267.

30. La Mura V, Pasarín M, Rodriguez-Vilarrupla A, García-Pagán JC, Bosch J, Abraldes JG. Liver sinusoidal endothelial dysfunction after LPS administration: a role for inducible-nitric oxide synthase. J Hepatol 2014;61:1321-1327.

31. Wells RG, Schwabe R. Origin and function of myofibroblasts in the liver. Semin Liver Dis 2015;35:97-106.

32. Wells RG. The portal fibroblast: not just a poor man's stellate cell. Gastroenterology 2014;147:41-47.

33. Han YP, Yan C, Zhou L, Qin L, Tsukamoto H. A matrix metalloproteinase- 9 activation cascade by hepatic stellate cells in transdifferentiation in the three-dimensional extracellular matrix. J Biol Chem 2007;282:12928-12939.

34. Li H, Mittal A, Makonchuk DY, Bhatnagar S, Kumar A. Matrix metal- loproteinase-9 inhibition ameliorates pathogenesis and improves skeletal muscle regeneration in muscular dystrophy. Hum Mol Genet 2009;18:2584-2598.

35. Gieling RG, Wallace K, Han YP. Interleukin-1 participates in the progression from liver injury to fibrosis. Am J Physiol Gastrointest Liver Physiol 2009;296:G1324-G1331.

36. Tchougounova E, Lundequist A, Fajardo I, Winberg JO, Abrink M, Pejler G. A key role for mast cell chymase in the activation of promatrix metalloprotease-9 and pro-matrix metalloprotease-2. J Biol Chem 2005;280:9291-9296.

37. Nathan C. Is iNOS beginning to smoke? Cell 2011;147:257-258.

38. Stahl W, Matejovic M, Radermacher P. Inhibition of nitric oxide synthase during sepsis: revival because of isoform selectivity? Shock 2010;34:321-322.

39. Cohen-Naftaly M, Friedman SL. Current status of novel antifibrotic therapies in patients with chronic liver disease. Therap Adv Gastroenterol 2011;4:391-417.

40. Levesque MC, Hobbs MR, O'Loughlin CW, Chancellor JA, Chen Y, Tkachuk AN, et al. Malaria severity and human nitric oxide synthase type 2 (NOS2) promoter haplotypes. Hum Genet 2010;127:163-182.

41. Hobbs MR, Udhayakumar V, Levesque MC, Booth J, Roberts JM, Tkachuk AN, et al. A new NOS2 promoter polymorphism associated with increased nitric oxide production and protection from severe malaria in Tanzanian and Kenyan children. Lancet 2002;360:14681475.

42. Kun JF, Mordmüller B, Perkins DJ, May J, Mercereau-Puijalon O, Alpers $\mathrm{M}$, et al. Nitric oxide synthase 2(Lambaréné) (G-954C), increased nitric oxide production, and protection against malaria. J Infect Dis 2001;184:330-336.

43. Holla LI, Stejskalova A, Znojil V, Vasku A. Analysis of the inducible nitric oxide synthase gene polymorphisms in Czech patients with atopic diseases. Clin Exp Allergy 2006;36:1592-1601.

44. Bugeja MJ, Booth DR, Bennetts BH, Heard RN, Burgner D, Stewart GJ. An investigation of NOS2A promoter polymorphisms in Australian multiple sclerosis patients. Eur J Hum Genet 2005;13:815-822.

45. Fu L, Zhao Y, Lu J, Shi J, Li C, Liu H, et al. Functional single nucleotide polymorphism-1026C/A of inducible nitric oxide synthase gene with increased YY1-binding affinity is associated with hypertension in a Chinese Han population. J Hypertens 2009;27:991-1000.

46. Yoneda M, Hotta K, Nozaki Y, Endo H, Tomeno W, Watanabe S, et al. Influence of inducible nitric oxide synthase polymorphisms in Japanese patients with non-alcoholic fatty liver disease. Hepatol Res 2009;39:963-971.

47. Tanabe K, Taura K, Koyama Y, Yamamoto G, Nishio T, Okuda Y, et al. Migration of splenic lymphocytes promotes liver fibrosis through modification of T helper cytokine balance in mice. J Gastroenterol 2015;50:1054-1068.

48. Guo Z, Geller DA. microRNA and human inducible nitric oxide syn- 
thase. Vitam Horm 2014;96:19-27.

49. Iwakiri Y. S-nitrosylation of proteins: a new insight into endothelial cell function regulated by eNOS-derived NO. Nitric Oxide 2011;25:95-101.

50. Iwakiri Y, Satoh A, Chatterjee S, Toomre DK, Chalouni CM, Fulton D, et al. Nitric oxide synthase generates nitric oxide locally to regulate compartmentalized protein S-nitrosylation and protein trafficking. Proc Natl Acad Sci U S A 2006;103:19777-19782.
51. Sangwung P, Greco TM, Wang Y, Ischiropoulos H, Sessa WC, Iwakiri Y. Proteomic identification of S-nitrosylated Golgi proteins: new insights into endothelial cell regulation by eNOS-derived NO. PLOS One 2012;7:e31564.

52. Stamler JS, Simon DI, Osborne JA, Mullins ME, Jaraki O, Michel T, et al. S-nitrosylation of proteins with nitric oxide: synthesis and characterization of biologically active compounds. Proc Natl Acad Sci U S A 1992;89:444-448. 